\title{
Pseudoachondroplasia: Report on a South African family
}

\author{
S Moosa, ${ }^{1} \mathrm{MB} B C h, \mathrm{DCH}(\mathrm{SA})$; G Nishimura, ${ }^{2} \mathrm{MD}$
}

${ }^{1}$ Division of Human Genetics, National Health Laboratory Service and School of Pathology, University of the Witwatersrand, Johannesburg, South Africa ${ }^{2}$ Department of Pediatric Imaging, Tokyo Metropolitan Children's Medical Center, Tokyo, Japan

Corresponding author: S Moosa (shahidamoosa@gmail.com)

Pseudoachondroplasia is an autosomal dominant skeletal dysplasia that results in disproportionately short stature, severe brachydactyly with strikingly lax small joints, malalignments of the lower limbs, and characteristic radiological features. Although named 'false achondroplasia', the entity is a distinct condition, in which affected individuals are born with normal length and have a normal facies, but is often only recognised after the age of 2 years, when the disproportion and waddling gait become evident. We report on an affected South African father and daughter, and highlight their clinical and radiographic features.

S Afr J Rad 2013;17(2):65-67. DOI:10.7196/SAJR.767

Pseudoachondroplasia (OMIM 177170) is an autosomal dominant skeletal dysplasia that results in disproportionately (micromelic) short stature, severe brachydactyly with strikingly lax small joints, various malalignments of the lower limbs owing to laxity of the large joints, and characteristic radiological features. Although named 'false achondroplasia, the entity is a distinct condition in which affected individuals are born with normal length and have a normal facies. It is often only recognised after the age of 2 years, when the disproportion and waddling gait become evident. The radiological manifestations are descriptively termed spondylo-epi-metaphyseal dysplasia (SEMD). Significant retardation of epiphyseal ossification manifests with small, round epiphyses. The epiphyseal dysplasia leads to premature degenerative joint disease. The metaphyses are irregularly ossified. The vertebral bodies appear mildly flat with biconvex endplates. The distinctive vertebral anomalies, apparent in pre-pubertal individuals, often resolve with age, thus emphasising the importance of correct diagnosis in childhood. We present a South African (SA) family with an affected father and daughter, and highlight their clinical and radiographic features.

\section{Clinical features}

The proband was a 12-year-old girl who presented with short stature and painful knees and ankles (Fig. 1). She was reported to have normal birth length and was of average intelligence. Her height was $104.5 \mathrm{~cm}$ ( $<<3$ rd centile), and her upper-to-lower segment ratio 1.6 (increased) and arm span was $92.5 \mathrm{~cm}$ (decreased). Weight and head circumference were normal. She stood with an exaggerated lumbar lordosis and had a waddling gait. She had no facial dysmorphic features. The upper limbs showed shortening of all segments with prominent joints of the elbow and wrists. The elbow joints were restricted, while the wrist and finger joints showed hyperlaxity. Brachydactyly with squared fingertips and deepset nails was found. The lower limbs showed marked genu varus, shortening of all limb segments and flat, short feet. Mild lumbar scoliosis was evident.
Her 48-year-old father was found to have a very similar phenotype (Fig. 2). He presented with difficulty in walking and with painful knee and hip joints. Genu valgus $(\mathrm{L}>\mathrm{R})$ was noted, and all joints were stiff. His face, upper limbs and feet resembled those of his daughter. His height was $125.5 \mathrm{~cm}(<<3 \mathrm{rd}$ centile), with a decreased arm span and increased upper-to-lower segment ratio.

\section{Radiographic features}

Radiographs of these two affected individuals showed skeletal changes involving the spine, metaphyses and epiphyses of tubular bones descriptively termed SEMD (Figs 3 - 7). The proband exhibited biconvex deformity with anterior beaking of the vertebral bodies, but platyspondyly was not evident. Mild thoracolumbar gibbus was noted, with hypoplasia of the first vertebral body (Fig. 3). The ilia were somewhat hypoplastic along with mildly short greater sciatic notches and steep, irregular acetabulae. The proximal femoral epiphyses were small and round. Ossification of the greater trochanters was irregular (Fig. 6). The epiphyses of other long bones were also small and irregular in ossification. The metaphyses of the long bones were flared and irregular. Spur-like projections were noted in some of the metaphyseal margins, particularly of the proximal tibiae (Figs 5 and 6). Carpal bones showed delayed ossification and irregular margins. The short tubular bones showed significant shortening along with epiphyseal irregularities (Fig. 7). Spinal radiographs of the father demonstrated thoracolumbar scoliosis with mild modification of the vertebral bodies (Fig. 4).

\section{Discussion}

Pseudoachondroplasia was first described by Maroteaux and Lamy in 1959. ${ }^{[1]}$ As the most common of the SEMD group of disorders, it is estimated to affect 1 in every 20000 individuals. ${ }^{[2]}$ Typically, affected individuals have a normal birth length and no facial dysmorphic features. Joint laxity, particularly of the small joints of the hand, is another clinical hallmark. As in this family, joint pain is a common presenting symptom. The lower limbs may show genu valgus, varus or a windswept deformity. 


\section{PICTORIAL ESSAY}

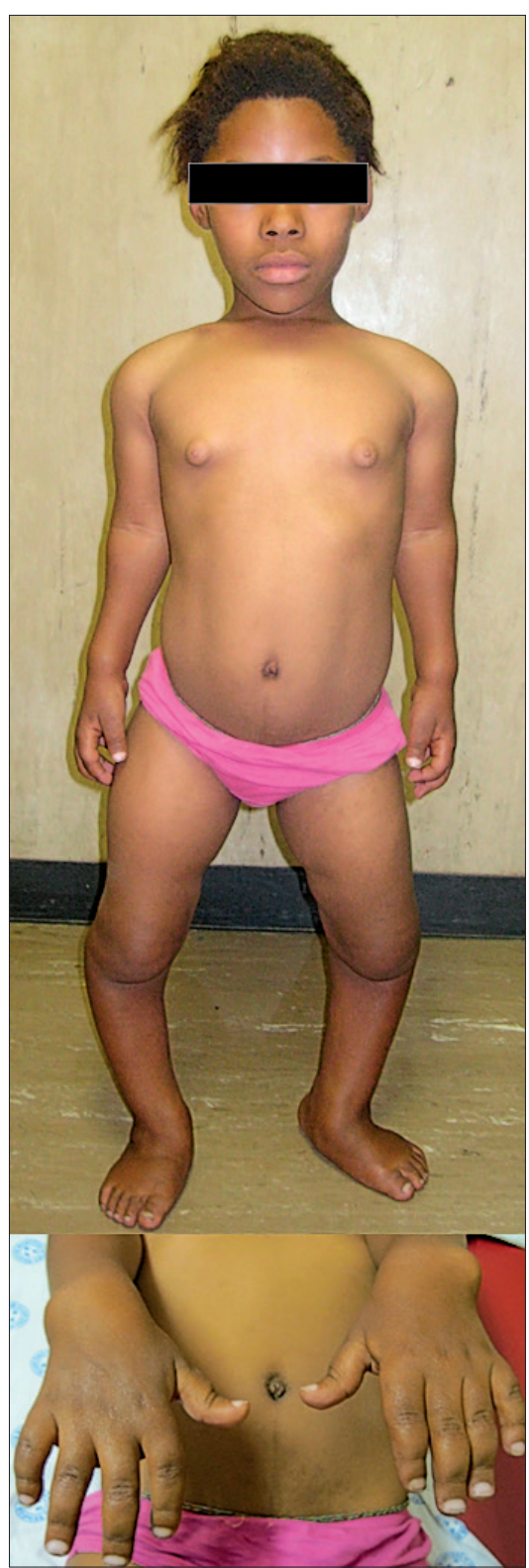

Fig. 1. Clinical photograph of the proband showing her disproportionate short stature, lack of facial dysmorphic features, marked genu varus and significant brachydactyly.

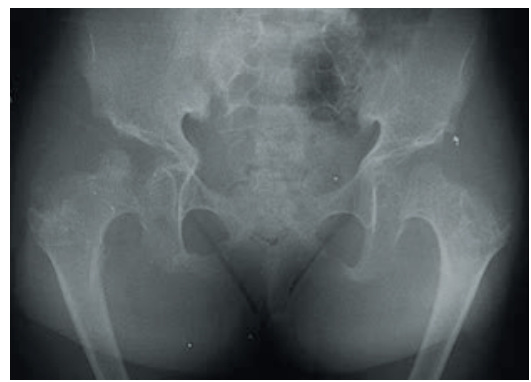

Fig. 6. Proband's pelvic X-ray (AP) shows small capital femoral epiphyses, short femoral necks, and irregular flared femoral metaphyseal borders and poorly modelled acetabulae with irregular margins.

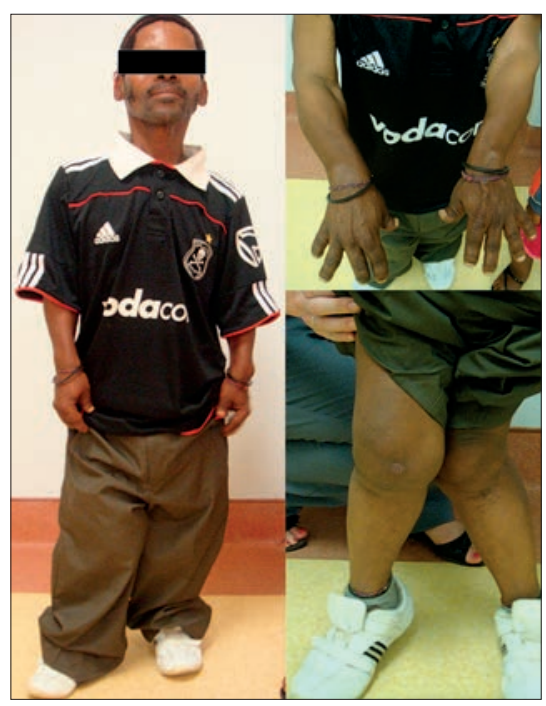

Fig. 2. Clinical photograph of the proband's father showing similar clinical phenotype, normal facies, genu valgus deformities and brachydactyly.

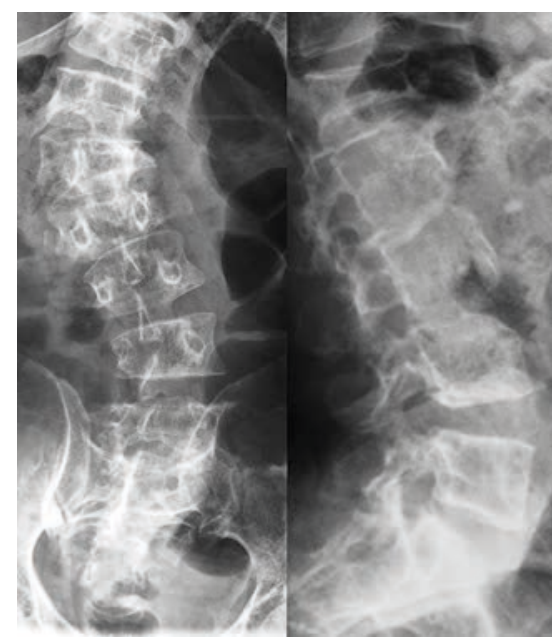

Fig. 4. Spine X-rays (AP and lateral) of the proband's father show marked scoliosis, degenerative changes, platyspondyly (probably secondary to compression fractures) and less beaking and tonguing of anterior vertebral bodies than in the proband.

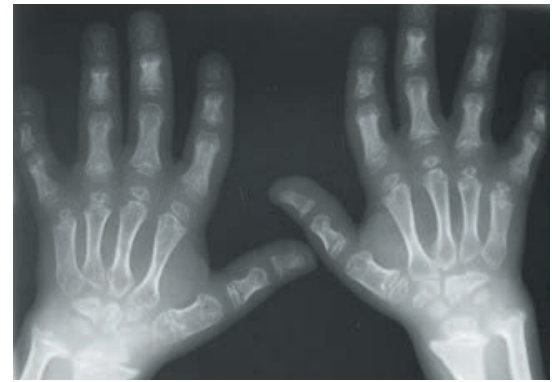

Fig. 7. Proband's AP hand X-ray shows significant brachydactyly, short metacarpals and phalanges with irregular metaphyses, and small, irregular carpal bones.

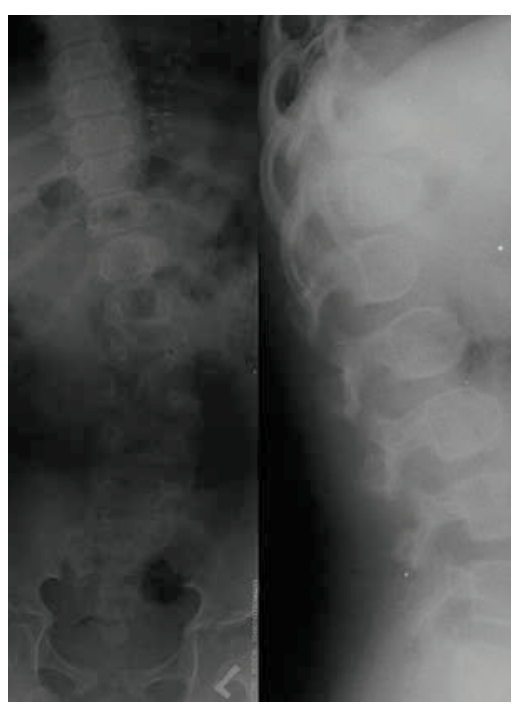

Fig. 3. Spine X-rays (AP and lateral) of the proband show mild scoliosis, anterior beaking and tonguing of vertebral bodies.

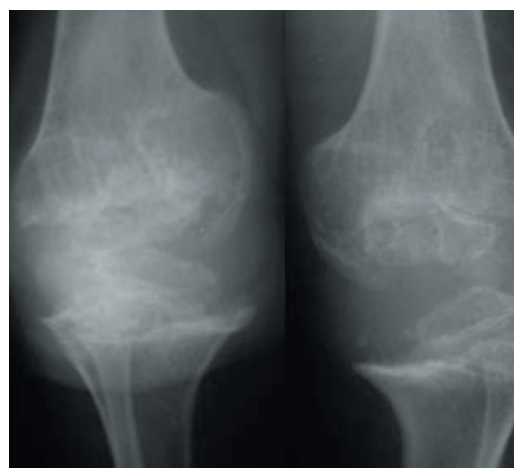

Fig. 5. Proband's knee X-rays (AP) show significant metaphyseal irregularities (flaring and splaying) and small epiphyses. 
The characteristic radiographic features include: (i) delayed epiphyseal ossification and irregular metaphyses of the long bones with small capital femoral epiphyses, short femoral necks and irregular, flared metaphyseal borders, and small pelvis and poorly modelled acetabulae with irregular margins that may be sclerotic, especially in older individuals; (ii) significant brachydactyly, particularly short metacarpals and phalanges with irregular metaphyses and small, irregular carpal bones; and (iii) anterior beaking or tonguing of the vertebral bodies, biconvex vertebral endplates, and platyspondyly in childhood. This distinctive appearance of the vertebrae normalises with age, emphasising the importance of obtaining the necessary images in childhood, to aid in making a definitive diagnosis.

The proband was 12 years old at the time that the accompanying radiographs were taken, and the platyspondyly had already resolved. Unfortunately, no earlier spinal images were available. The natural history of pseudoachondroplasia includes osteoarthritic changes of the extremities and the spine, which are related to abnormal epiphyseal development, along with joint laxity, and may occur in early adult life. Spinal osteoarthropathy may be associated with scoliosis. Degenerative joint disease is progressive; approximately half of all affected individuals eventually require hip replacement surgery. ${ }^{[3]}$

Pseudoachondroplasia is inherited in an autosomal dominant manner. Therefore, an affected individual has a $50 \%$ chance at every pregnancy of passing the condition on to the next generation. Mutations in the COMP gene, which encodes the cartilage oligomeric matrix protein, cause pseudoachondroplasia. This protein is a normal constituent of the extracellular matrix in cartilage, ligaments and tendons. When the gene is mutated, an abnormal accumulation of proteoglycans within cartilage cells occurs, which leads to early death of these cells and ultimately to defective bone growth and modelling. ${ }^{[2]}$

As molecular genetic testing is not yet available in SA, the diagnosis remains clinicoradiological. Ongoing care of affected individuals requires the participation of a multidisciplinary team to address their joint pain, limb deformities, scoliosis and psychosocial issues related to short stature. Timeous referral for evaluation by a medical geneticist is invaluable in confirming the diagnosis, providing genetic counselling to affected families and directing future management.

1. Maroteaux, P, Lamy M. Les formes pseudo-achondroplastiques des dysplasies spondylo-epiphysaires. Presse Med 1959;67:383386.

2. Tufan AC, Satiroglu-Tufan NL, Jackson GC, Semerci CN, Solak S, Yagci B. Serum or plasma cartilage oligomeric matrix protein concentration as a diagnostic marker in pseudoachondroplasia: Differential diagnosis of a family. Eur J Hum Genet 2007;15:1023-1028.

3. Online Mendelian Inheritance in Man. MIM Number: 177170. Baltimore, USA: Johns Hopkins University, 13 April 2010. http://omim.org (accessed 16 June 2012). 